\title{
The efficacy of continuous versus single- injection femoral nerve block in Total knee Arthroplasty: a systematic review and meta- analysis
}

Hsuan-Hsiao Ma ${ }^{1,2}$, Te-Feng Arthur Chou ${ }^{1,2}$, Shang-Wen Tsai ${ }^{1,2}$, Cheng-Fong Chen ${ }^{1,2^{*}}$, Po-Kuei Wu ${ }^{1,2}$ and Wei-Ming Chen ${ }^{1,2}$

\begin{abstract}
Background: Continuous femoral nerve block (CFNB) has been developed to extend the analgesic effect since the efficacy of single-injection femoral nerve block (sFNB) is often limited to approximately $16-24 \mathrm{~h}$. The aim of this meta-analysis was to validate the add-on effect of cFNB in the setting of a multimodal analgesic protocol.

Methods: We performed a comprehensive literature review on Web of Science, Embase, the Cochrane Library and PubMed. Eight randomized controlled trials $(N=626)$ that compared the efficacy of cFNB with sFNB were included. The primary outcome domains consist of visual analog scale (VAS) score at postoperative 24 and $48 \mathrm{~h}$. The secondary outcome domains include opioid consumption, length of hospital stay and incidence of nausea.

Results: Our analysis revealed that CFNB was associated with a lower VAS score at $24 \mathrm{~h}$ (SMD: $-0.277 ; 95 \% \mathrm{Cl}-0.503$ to -0.05$)$. However, the difference of VAS score did not meet the minimal clinically importance difference for total knee arthroplasty (TKA). VAS score at $48 \mathrm{~h}$ was similar between the CFNB and SFNB group. The cFNB group was associated with less amount of opioids consumed at both 24(SMD: $-1.056 ; 95 \% \mathrm{Cl}-1.737$ to -0.375$)$ and $48 \mathrm{~h}(\mathrm{SMD}$ : $-1.040 ; 95 \% \mathrm{Cl}-1.790$ to -0.289$)$. Length of hospital stay and incidence of nausea were similar between the two groups.
\end{abstract}

Conclusion: In the setting of a multimodal analgesic protocol, patients might benefit from cFNB with regards to a reduced need of opioids in the early postoperative period. However, we did not find a clinically significant difference in pain scores at different time points between the CFNB and sFNB group.

Level of evidence: I; meta-analysis.

Keywords: Continuous, Femoral nerve block, Nerve block, Pain, Single-injection, Total knee arthroplasty

\section{Background}

Postoperative pain management is one of the key components in the enhanced recovery after surgery (ERAS) pathway for total knee arthroplasty (TKA) [1]. To manage postoperative pain and facilitate early mobilization and rehabilitation, the concept of multimodal analgesic protocol has been employed [2-4]. Femoral nerve block

\footnotetext{
* Correspondence: swtsai.vghtpe@gmail.com

'Department of Orthopaedics and Traumatology, Taipei Veterans General Hospital, No. 201, Sec 2, Shi-Pai Road, Taipei 112, Taiwan

${ }^{2}$ Department of Orthopaedics, School of Medicine, National Yang-Ming University, Taipei, Taiwan
}

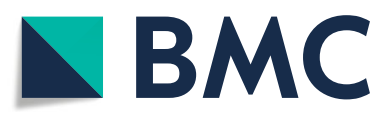

(c) The Author(s). 2020 Open Access This article is distributed under the terms of the Creative Commons Attribution 4.0 International License (http://creativecommons.org/licenses/by/4.0/), which permits unrestricted use, distribution, and reproduction in any medium, provided you give appropriate credit to the original author(s) and the source, provide a link to the Creative Commons license, and indicate if changes were made. The Creative Commons Public Domain Dedication waiver (http://creativecommons.org/publicdomain/zero/1.0/) applies to the data made available in this article, unless otherwise stated. TKAs as part of a multimedal ar Currently, there are two commonly used methods to administer femoral nerve blocks, the continuous femoral nerve block (cFNB) and single-shot femoral nerve block (sFNB). FNB has been proven to have a beneficial effect on pain management but it remains uncertain whether cFNB can lead to additional benefits compared with that of sFNB in the setting of multimodal analgesic protocols (eg. pain scores at different time points, amount of opioid consumption, adverse effects of opioids, length of hospital stay and functional outcomes) [9-14]. Due to 
the mixed results in current literature, it is undetermined whether the additional benefits of cFNB over sFNB are worthy of its extra cost and time involved. Therefore, the aim of this meta-analysis was to determine the efficacy of cFNB compared with the sFNB group and whether cFNB is a superior postoperative pain management modality in patients who had undergone TKA.

\section{Methods}

\section{Search strategy}

We followed the statement on preferred reporting items for systematic reviews and meta-analysis (PRISMA) guidelines (Table 1) and searched a comprehensive search on databases including PubMed, Embase, the Cochrane Library and Web of Science from the earliest record to September 2019. The following searching strategy was similar to the article we have published before [15]. We reviewed all of the articles that discussed continuous femoral nerve block (cFNB) versus singleinjection femoral nerve block (sFNB) in TKA. Prior to analysis, we reviewed all included studies that evaluated cFNB or sFNB for the relief of postoperative pain in patients who had received TKA surgery and excluded studies not written in English or when the full article was unavailable. The keywords were with the following combinations: (femoral nerve block OR regional anesthesia OR continuous femoral nerve block OR nerve block) AND (total knee arthroplasty OR total knee replacement). We included only RCTs and excluded comparative experimental trials, single-armed follow-up studies, case series and case reports. All the included studies comprised at least two arms: cFNB and sFNB.

\section{Eligibility criteria}

The included trials (1)enrolled patients who had received unilateral TKA (2) randomized them to receive intervention of cFNB as the pain control method for TKA or sFNB as the primary method to manage pain (3) compared outcome parameters of visual analog scale (VAS) at postoperative 24 and $48 \mathrm{~h}$, cumulative dose of opioids consumption at postoperative 24 and $48 \mathrm{~h}$, length of hospital stay and incidence of postoperative nausea (4)conducted follow-up rate at least $80 \%$ and included 1 of the above outcome parameters.

\section{Data extraction and quality assessment}

A pair of reviewers independently abstracted each identified article. The included data were study, patient characteristics, enrolled sample number, type of treatment arms, anesthesia method, regimen of the first bolus in $\mathrm{sFNB} / \mathrm{cFNB}$, regimen and rate of infusion in cFNB. The outcome parameters were pain, cumulative dose of opioids consumption, length of hospital stay and incidence of nausea after the surgery were listed in Table 1.

\section{Data synthesis and analysis}

Continuous outcome measures such as postoperative VAS score at different time, the cumulative dose of opioids consumed at postoperative 24 and $48 \mathrm{~h}$, length of hospital stay were pooled and standardized mean differences (SMDs) were calculated. Negative SMD values

Table 1 Characteristics of included studies

\begin{tabular}{|c|c|c|c|c|c|c|c|c|c|c|c|c|}
\hline \multirow[t]{2}{*}{$\begin{array}{l}\text { Year/First } \\
\text { author }\end{array}$} & \multirow[t]{2}{*}{$\begin{array}{l}\text { Study } \\
\text { design }\end{array}$} & \multirow[t]{2}{*}{$\begin{array}{l}\text { Enrolled Sample } \\
\text { number (G1/G2) }\end{array}$} & \multirow[t]{2}{*}{ Comparing } & \multirow[t]{2}{*}{ Anesthesia } & \multirow[t]{2}{*}{$\begin{array}{l}\text { Regimen of first bolus } \\
\text { in sFNB/CFNB }\end{array}$} & \multirow[t]{2}{*}{$\begin{array}{l}\text { Regimen and rate } \\
\text { of infusion in CFNB }\end{array}$} & \multicolumn{6}{|c|}{$\begin{array}{l}\text { Outcome } \\
\text { measurement }\end{array}$} \\
\hline & & & & & & & $a$ & $\mathrm{~b}$ & C & $d$ & $\mathrm{e}$ & $f$ \\
\hline 2019 Angers & $\mathrm{RCT}$ & $45 / 45$ & cFNB, sFNB & SA/GA & 20 ml, 0.05\% Ropi & $0.15 \%$ Ropi, $7 \mathrm{ml} / \mathrm{hr}$ & & & & & V & \\
\hline 2018 Dixit & $\mathrm{RCT}$ & $44 / 41$ & CFNB, sFNB & SA & $20-25 \mathrm{ml}, 0.5 \%$ Ropi & $0.2 \%$ Ropi, rate not mentioned & V & V & V & V & V & V \\
\hline 2015 Wyatt & $\mathrm{RCT}$ & $42 / 42$ & cFNB, sFNB & SA/GA & 20 ml, 0.25\% Bupi & 0.125\% Bupi $10 \mathrm{ml} / \mathrm{hr}$ & V & V & & & V & \\
\hline 2013 Chan & $\mathrm{RCT}$ & $65 / 69$ & cFNB, sFNB & SA/GA & $\begin{array}{l}20 \mathrm{ml}, 0.25 \% \text { Bupi } \\
\text { with 1:400,000 Epi }\end{array}$ & $\begin{array}{l}0.125 \% \text { Bupi } \\
4 \mathrm{ml} / \mathrm{hr}\end{array}$ & V & V & V & V & V & V \\
\hline 2013 Albrecht & $\mathrm{RCT}$ & $60 / 33$ & cFNB, sFNB & SA & $\begin{array}{l}\text { sFNB: } 30 \mathrm{ml}, 0.375 \% \text { Ropi } \\
\text { with 1:400,000 Epi } \\
\text { cFNB: } 20 \mathrm{ml}, 0.2 \% \text { Ropi } \\
\text { with 1:400,000 Epi }\end{array}$ & $\begin{array}{l}\text { Ropi, } 10 \text { mg/hr. } \\
10 \text { mg bolus allowed } \\
\text { every } 30 \text { mins }\end{array}$ & V & V & V & V & & V \\
\hline 2010 Park & $\mathrm{RCT}$ & $60 / 20$ & $c F N B, s F N B$ & SA & $\begin{array}{l}20 \mathrm{ml}, 0.125 \% \text { Bupi } \\
\text { with 1:200,000 Epi }\end{array}$ & $\begin{array}{l}0.125 \% \text { Bupi } \\
2,4,6 \mathrm{ml} / \mathrm{hr}\end{array}$ & V & V & V & V & & \\
\hline 2006 Salinas & $\mathrm{RCT}$ & $18 / 18$ & cFNB, sFNB & SA & $\begin{array}{l}30 \mathrm{ml}, 0.375 \% \text { Ropi } \\
\text { with 1:400,000 Epi }\end{array}$ & $\begin{array}{l}0.2 \% \text { Ropi } \\
10 \mathrm{ml} / \mathrm{hr}\end{array}$ & V & V & V & V & V & \\
\hline 1996 Hirst & $\mathrm{RCT}$ & $11 / 11$ & cFNB, sFNB & GA & $\begin{array}{l}20 \mathrm{ml}, 0.5 \% \text { Bupi } \\
\text { with 1:200,000 Epi }\end{array}$ & $\begin{array}{l}0.125 \% \text { Bupi } \\
6 \mathrm{ml} / \mathrm{hr}\end{array}$ & V & V & V & V & & V \\
\hline
\end{tabular}

G1 group: study group (cFNB); G2 Group: control group (sFNB)

Outcome measure: $a=$ VAS at $24 \mathrm{~h} b=$ VAS at $48 \mathrm{~h}, \mathrm{c}=$ amount of analgesics consumed within $24 \mathrm{~h}, \mathrm{~d}=$ amount of analgesics consumed within $48 \mathrm{~h}$, e = Length of hospital stay, $\mathrm{f}=$ nausea or vomiting event

FNB femoral nerve block; SA spinal anesthesia; GA general anesthesia

Bupi Bupivacaine; Epi Epinephrine; Ropi Ropivacaine 


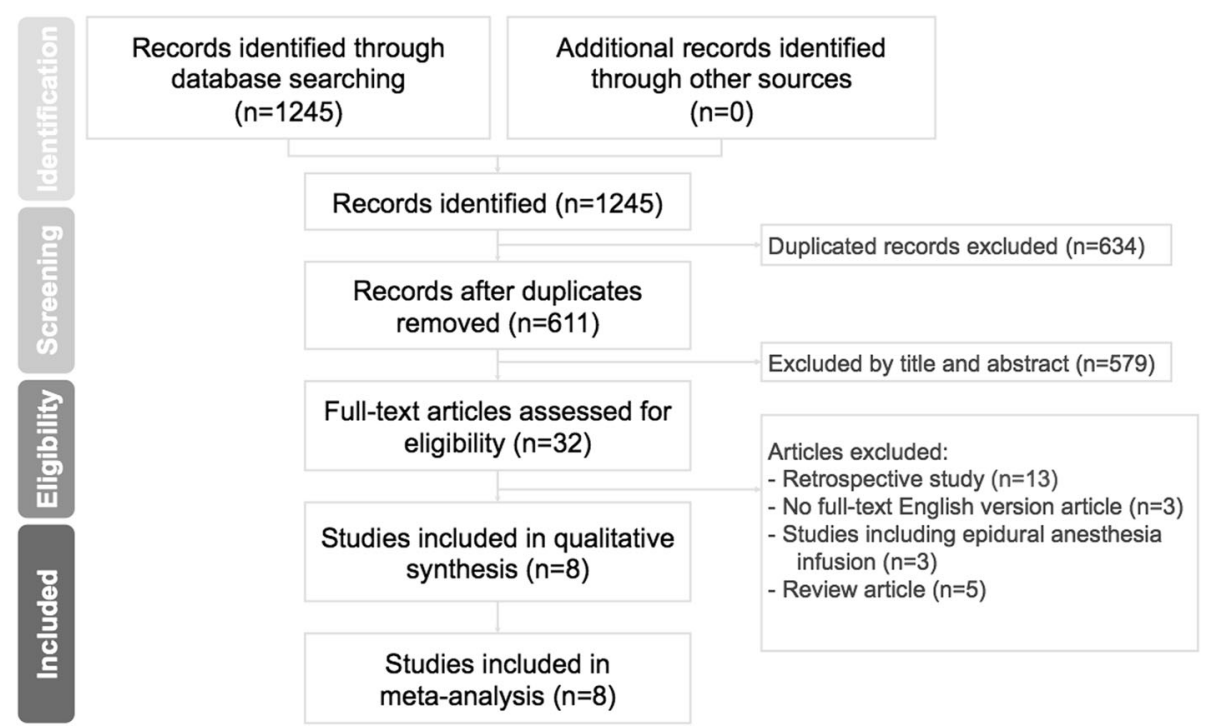

Fig. 1 Preferred reporting items for systematic reviews and meta-analysis (PRISMA) study flow diagram

indicated that cFNB was a more favorable treatment option. The incidence of nausea after surgery (assessed with odds ratio) were reported as binary outcomes. A random effect model was utilized to pool individual SMDs and ORs. Analyses were performed using Comprehensive Meta-Analysis (CMA) software, version 3 (Biostat, Englewood, NJ, USA). Between-trial heterogeneity was determined by using $I^{2}$ tests; values $>50 \%$ were regarded as considerable heterogeneity. Statistical significance was defined as $p$-values $<0.05$. Using the Cochrane tool for assessing risk of bias in randomized trials, a pair of reviewers independently evaluated each included study and documented their potential for selection bias, performance bias, detection bias, attrition bias, and reporting bias using. Funnel plots were constructed to visually detect the presence of publication bias.

\section{Results}

Of 1245 relevant articles identified, 634 duplicate records were removed. We further excluded 579 articles after reading titles and abstracts. Twenty-four studies were excluded after reading the full article, based on the inclusion criteria. There was agreement between reviewers at the full-text review stage and eight RCTs that compared the efficacy of cFNB with sFNB in TKA were included. (Fig. 1) Study details of all 8 studies are listed in Table 1.

\section{Outcomes for cFNB vs sFNB}

Pain relief assessed at postoperative 24 hours and $48 \mathrm{~h}$

Of 7 studies (a total of 534 patients), the VAS score at postoperative $24 \mathrm{~h}$. cFNB had lower VAS score at postoperative $24 \mathrm{~h}$ comparing with the sFNB group (SMD: $-0.277 ; 95 \% \mathrm{CI}-0.503$ to -0.05 ; heterogeneity: $\mathrm{I}^{2}=$

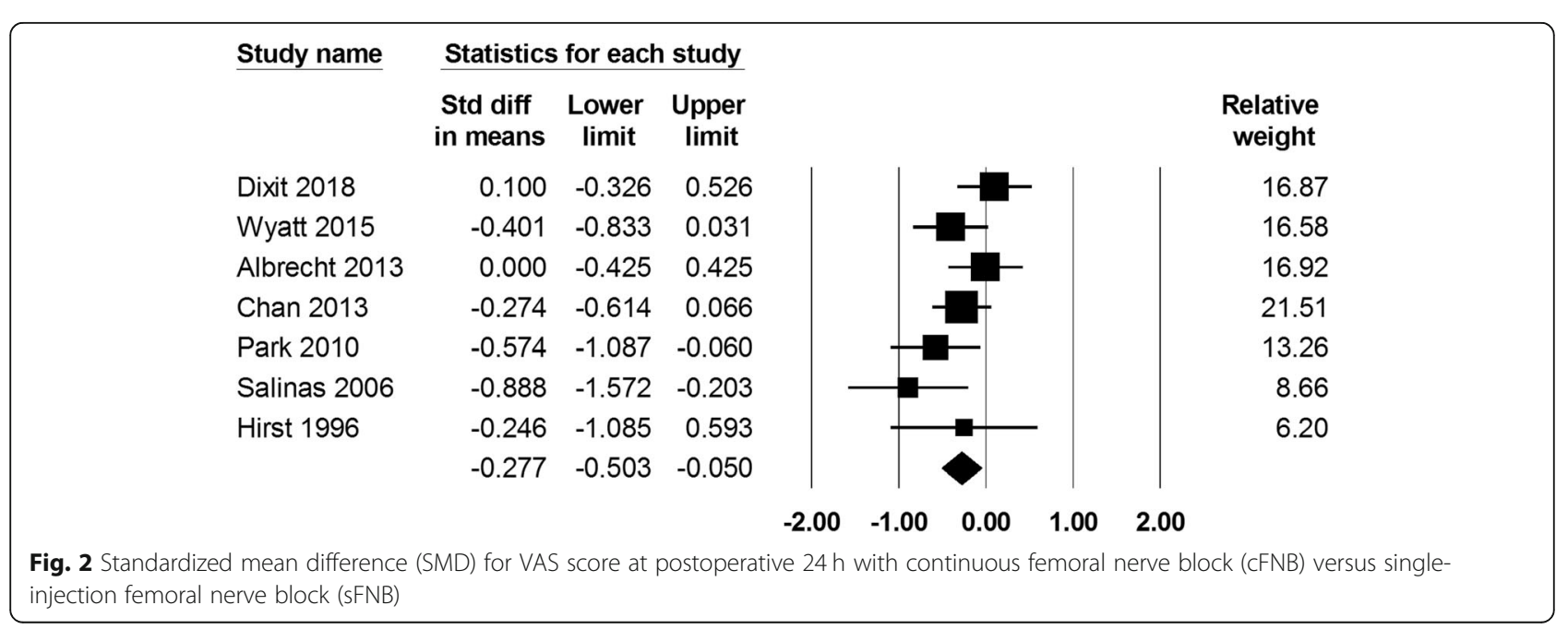




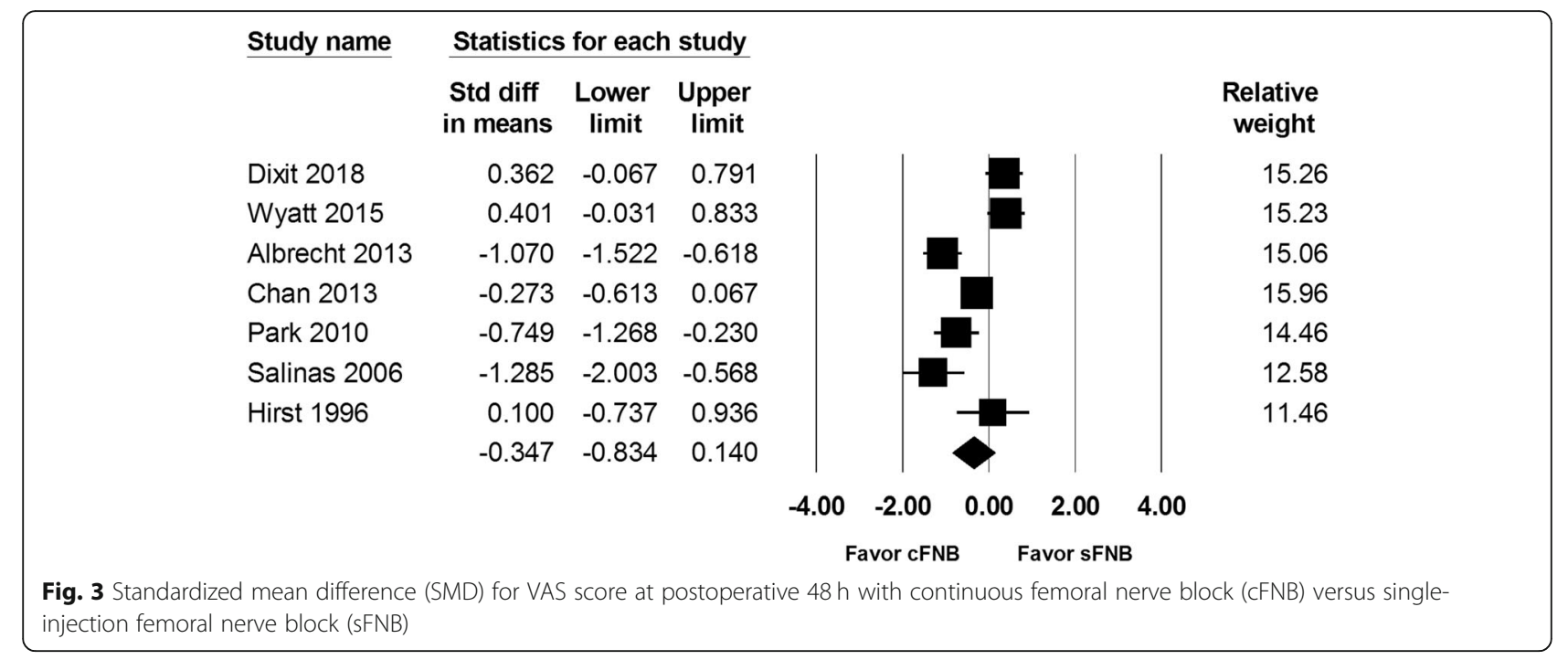

35.181; Fig. 2). VAS score at $24 \mathrm{~h}$ in the cFNB and sFNB group were $47.1 \mathrm{~mm}$ and $47.6 \mathrm{~mm}$, respectively. The difference in VAS scores between the two groups $(0.5 \mathrm{~mm})$ did not reach the minimal clinically important difference (MCID) for TKA, which was reported to be $16.1 \mathrm{~mm}$ [16].

The VAS score at postoperative $48 \mathrm{~h}$ was recorded in 7 studies (a total of 534 patients). VAS score at $48 \mathrm{~h}$ in the cFNB and sFNB group were $49.1 \mathrm{~mm}$ and $47.8 \mathrm{~mm}$, respectively. The VAS scores at $48 \mathrm{~h}$ were similar between the two groups (SMD: $-0.347 ; 95 \% \mathrm{CI}-0.834$ to 0.140; Heterogeneity: $\mathrm{I}^{2}=85.49$; Fig. 3).

Total amount of opioids consumed at postoperative 24 and 48 hours

Total amount of opioids consumed at postoperative $24 \mathrm{~h}$ and $48 \mathrm{~h}$ were reported in 6 studies and included 450 and 447 patients, respectively. The total amount of opioids used at postoperative $24 \mathrm{~h}$ in the cFNB and sFNB group were $16.6 \mathrm{mg}$ and $25.7 \mathrm{mg}$, respectively. At postoperative $48 \mathrm{~h}$, a total of $31.4 \mathrm{mg}$ and $42.1 \mathrm{mg}$ of opioids were consumed in the cFNB and sFNB group, respectively. The results showed that cFNB group was associated with a lower opioids consumption at both postoperative 24h (SMD: -1.056; 95\% CI -1.737 to 0.375; Heterogeneity: $\mathrm{I}^{2}=89.98$; Fig. 4) and $48 \mathrm{~h}$ (SMD: -1.040 ; $95 \%$ CI -1.790 to -0.289 ; Heterogeneity: $\mathrm{I}^{2}=$ 91.73; Fig. 5), compared with the sFNB group.

\section{Length of hospital stay}

Of 5 studies (a total of 429 patients), the length of hospital study showed no significant difference found between the cFNB and the sFNB group (SMD: -0.089; 95\% $\mathrm{CI}-0.279$ to 0.101; Heterogeneity: $\mathrm{I}^{2}=0.00$; Fig. 6).

Incidence of Postoperative Nausea.

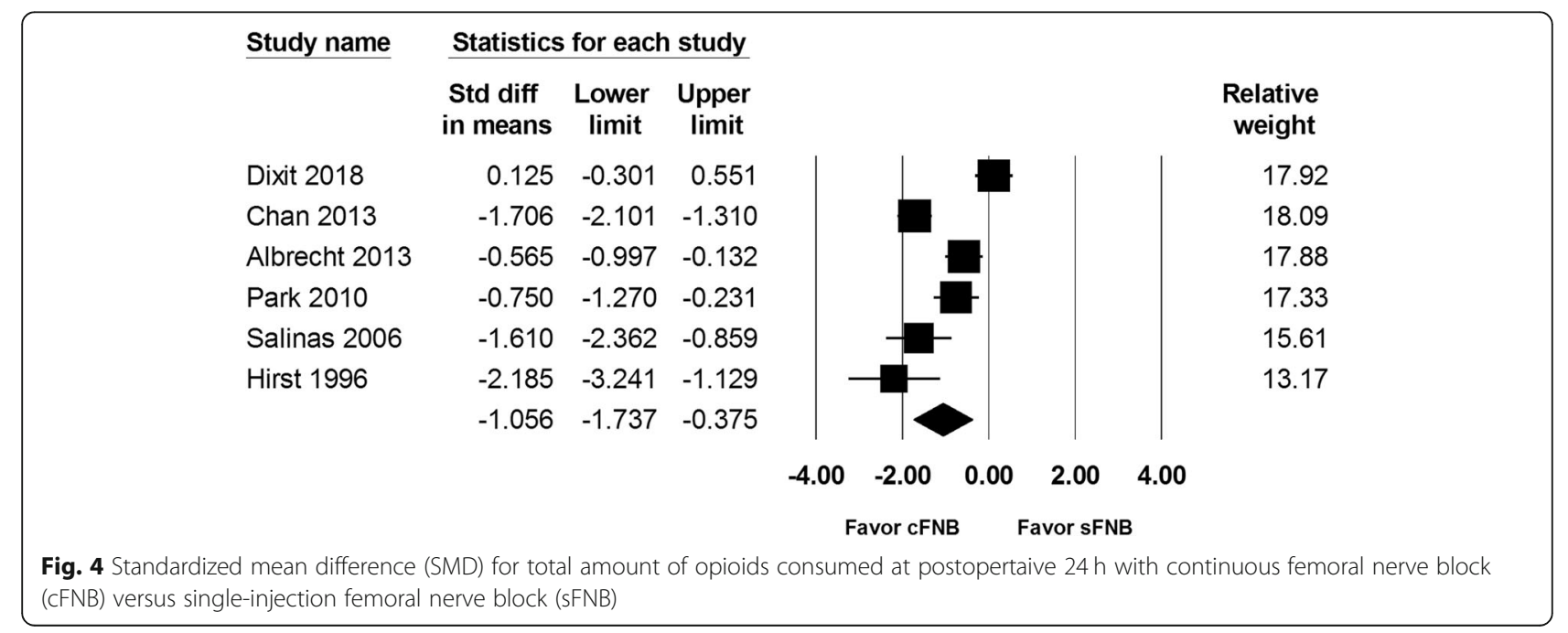




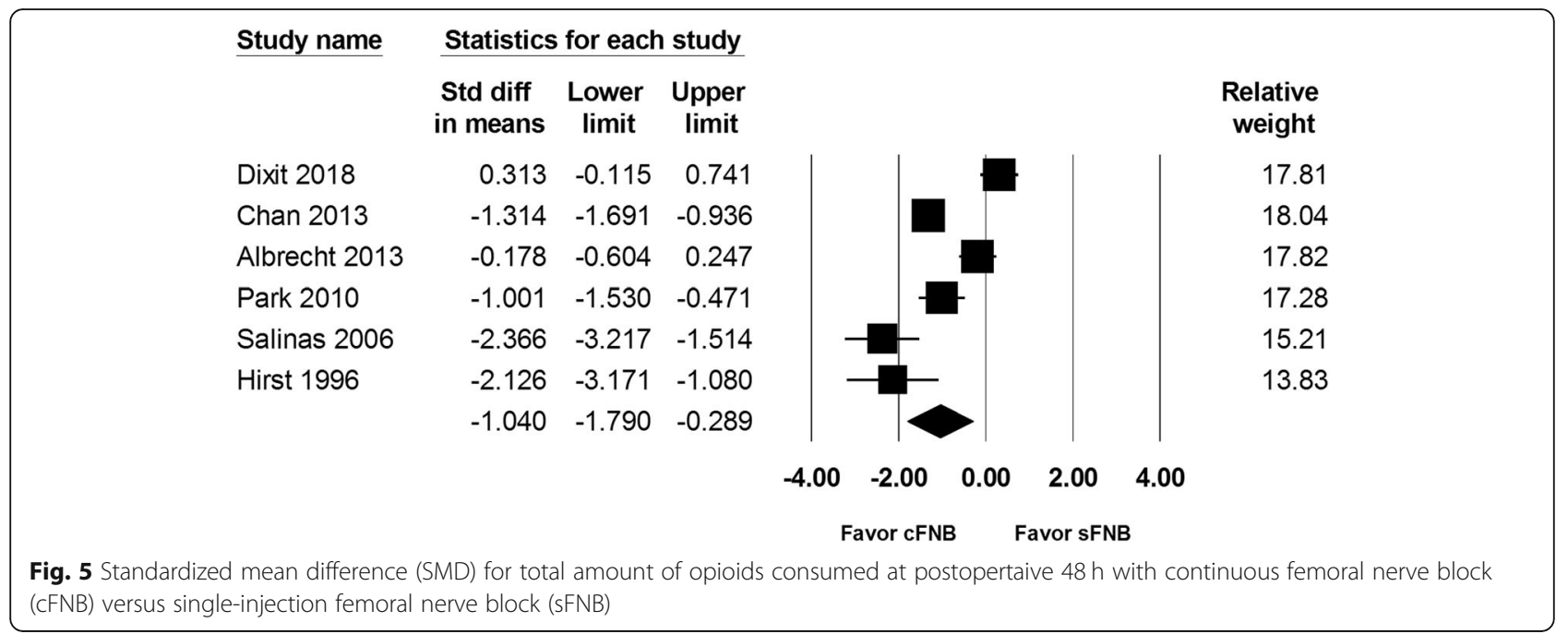

3 studies (334 patients) assessed postoperative nausea and the pooled data showed no significant difference between 2 group (OR: 0.667; 95\% CI 0.287 to 1.547 ; Heterogeneity: $\mathrm{I}^{2}=62.40$; Fig. 7 ).

\section{Risk of publication bias}

The risk of publication bias is shown in Fig. 8 and Fig. 9. The random sequence generation (selection bias) was unclear in 1 of the 8 (12.5\%) studies. The completeness of the reported data (reporting bias) was unclear in 3 of the $8(37.5 \%)$ studies. In Fig. S1-S6, we demonstrated the funnel plots for SMD and $\log$ odds ratio of all the outcome domains from every study.

\section{Discussion}

In this meta-analysis, we evaluated the efficacy of cFNB compared with that of sFNB in TKA. We included 8 studies with 626 patients. In comparison with the sFNB group, patients who received cFNB consumed less opioids at postoperative 24 and $48 \mathrm{~h}$. VAS score at $24 \mathrm{~h}$ was lower in the cFNB group but did not meet the MCID for TKA [16]. Other outcome parameters including VAS scores at $48 \mathrm{~h}$, length of hospital stay and postoperative nausea rates were not significant different.

Multimodal pain management in total joint arthroplasty involves a combination of modalities that act on different regions of the pain pathway to achieve better pain relief [2-4]. In the setting of multimodal analgesic protocols, a few studies reported a lower VAS score in the cFNB group [10,11,17], while other studies did not find a difference $[9,12,18,19]$. Since many studies employed spinal anesthesia (or subarachnoid block), PCA and/or sciatic nerve block as part of their regimen, it could have greatly alleviated pain and may masked the efficacy of femoral nerve blocks. Therefore, it appears that there was no significant difference between the cFNB and sFNB group [9-12, 14, 17]. Results from this meta-analysis showed a lower VAS score at $24 \mathrm{~h}$ in the

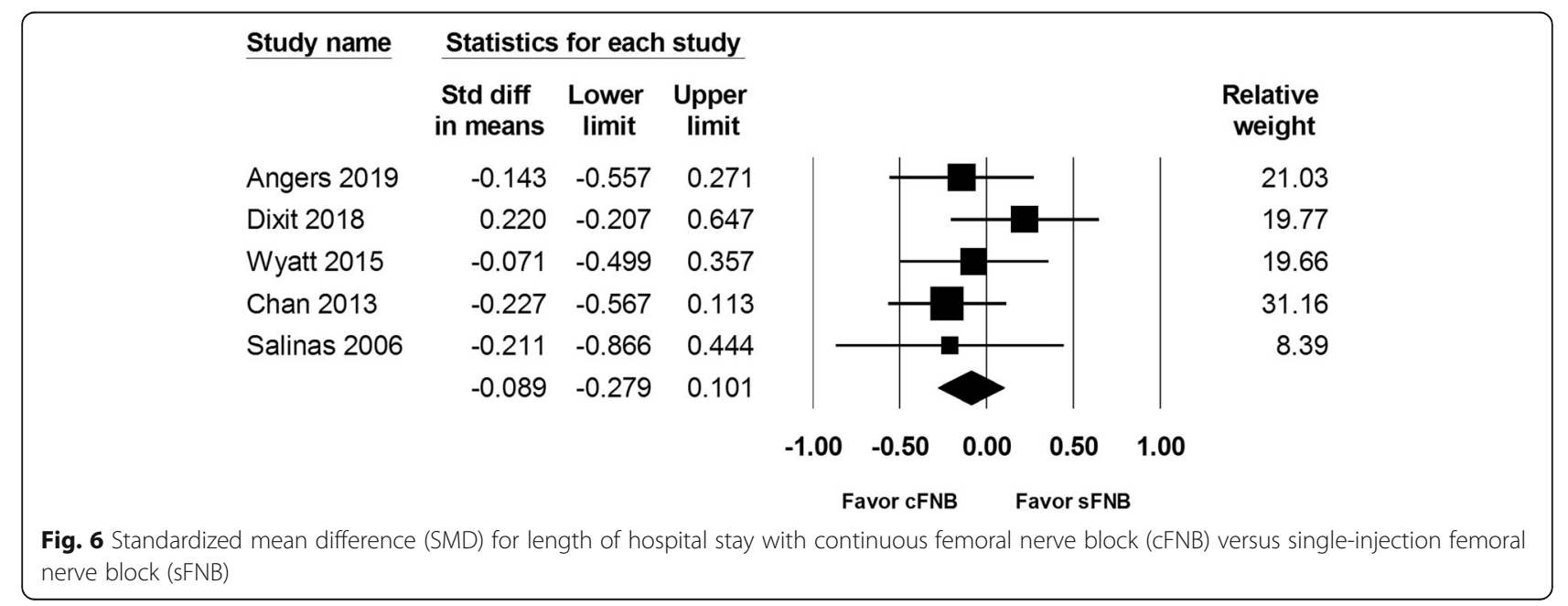




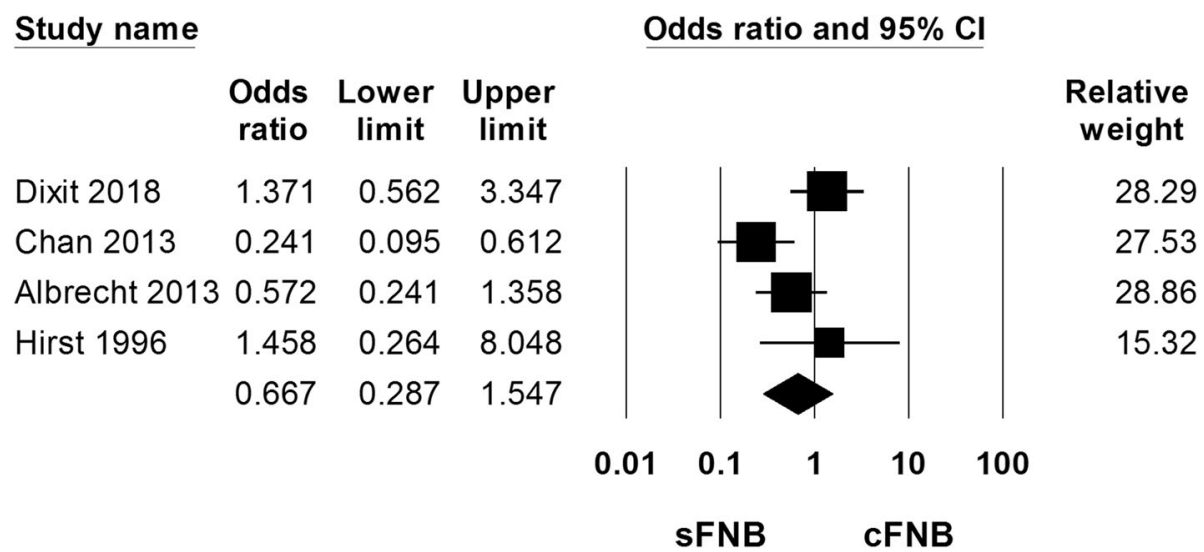

Fig. 7 Comparison of continuous femoral nerve block (CFNB) versus single-injection femoral nerve block (sFNB) with regard to postoperative nausea rate

cFNB group compared with the sFNB group. However, based on the MCID for TKA determined by Danoff et al., this difference did meet the criteria for clinical significance [16]. Since sFNB was reported to have an analgesic effect up to $24 \mathrm{~h}[20,21]$, cFNB was expected to extend the duration. However, the VAS score at $48 \mathrm{~h}$ was not different. The findings in pain scores is quite different from the results of meta-analysis conducted by Chan et al. in year 2014, in which the cFNB group was associated with a lower VAS score at both postoperative 24 and $48 \mathrm{~h} \mathrm{[13].} \mathrm{This} \mathrm{might} \mathrm{indicate} \mathrm{that} \mathrm{cFNB} \mathrm{has} \mathrm{lim-}$ ited add-on effect to sFNB with regards to pain scores in the setting of a multimodal analgesic protocol. In addition to assessing pain scores, consuming less amounts of opioids was another important issue that raises concerns [22-24]. We noted that patients can benefit from cFNB with regards to a reduced need of opioids in the early postoperative period ( 24 and $48 \mathrm{~h}$ ), compared with the sFNB group.

Another important aim of a pain management modality is to facilitate rehabilitation and functional recovery. A variety of outcome parameters have been evaluated and compared between cFNB and sFNB with mixed results [12, 14, 17-19]. Chan et al. compared the time required to achieve several functional performance goals including range of motion to $90^{\circ}$, active straight leg raise, the need for ambulating assistance with walking aids and obstacle clearance between cFNB and sFNB [12]. The authors did not find a significant difference in either of the parameters and concluded that cFNB and sFNB were similar in facilitating postoperative rehabilitation. In addition, Albrecht et al. also noted similar results in early functional recovery (distance walked, active and passive knee flexion) as well as long-term patient reported outcomes (WOMAC score and SF-36 score) between the two groups [17]. Similarly, Wyatt et al. found no differences in knee range of movement on postoperative day 1, 2 and 3 [19]. Dixit et al. found a higher proportion of patients in the cFNB group were able to walk independently at their rehabilitation session [18]. In contrast to those studies listed above that reported little or no benefits from $\mathrm{CFNB}$ with regards to functional outcome, Angers et al. noted that both sFNB and cFNB were associated with a detrimental effect on short- to

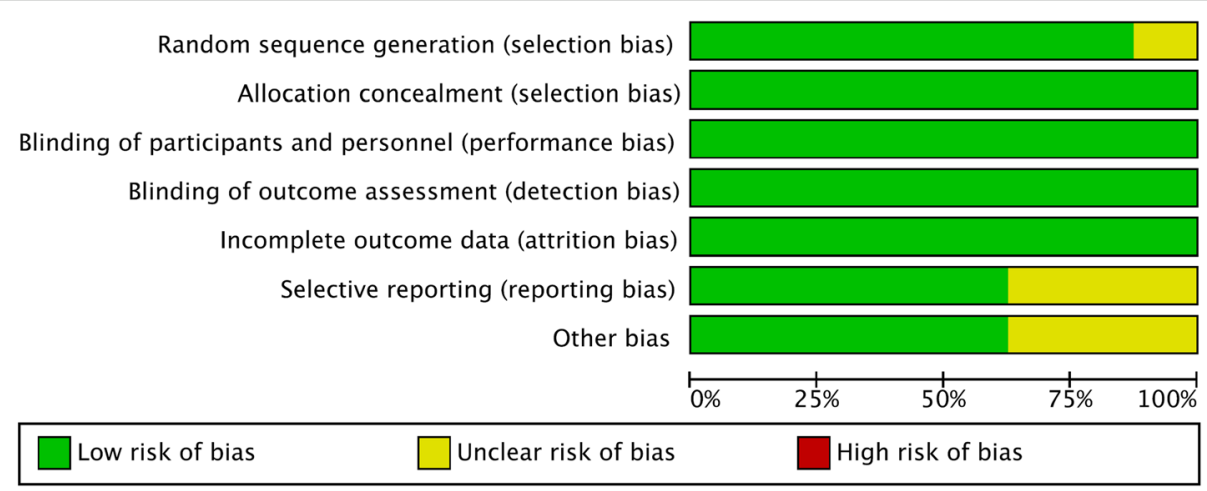

Fig. 8 Assessment of the risk of bias 


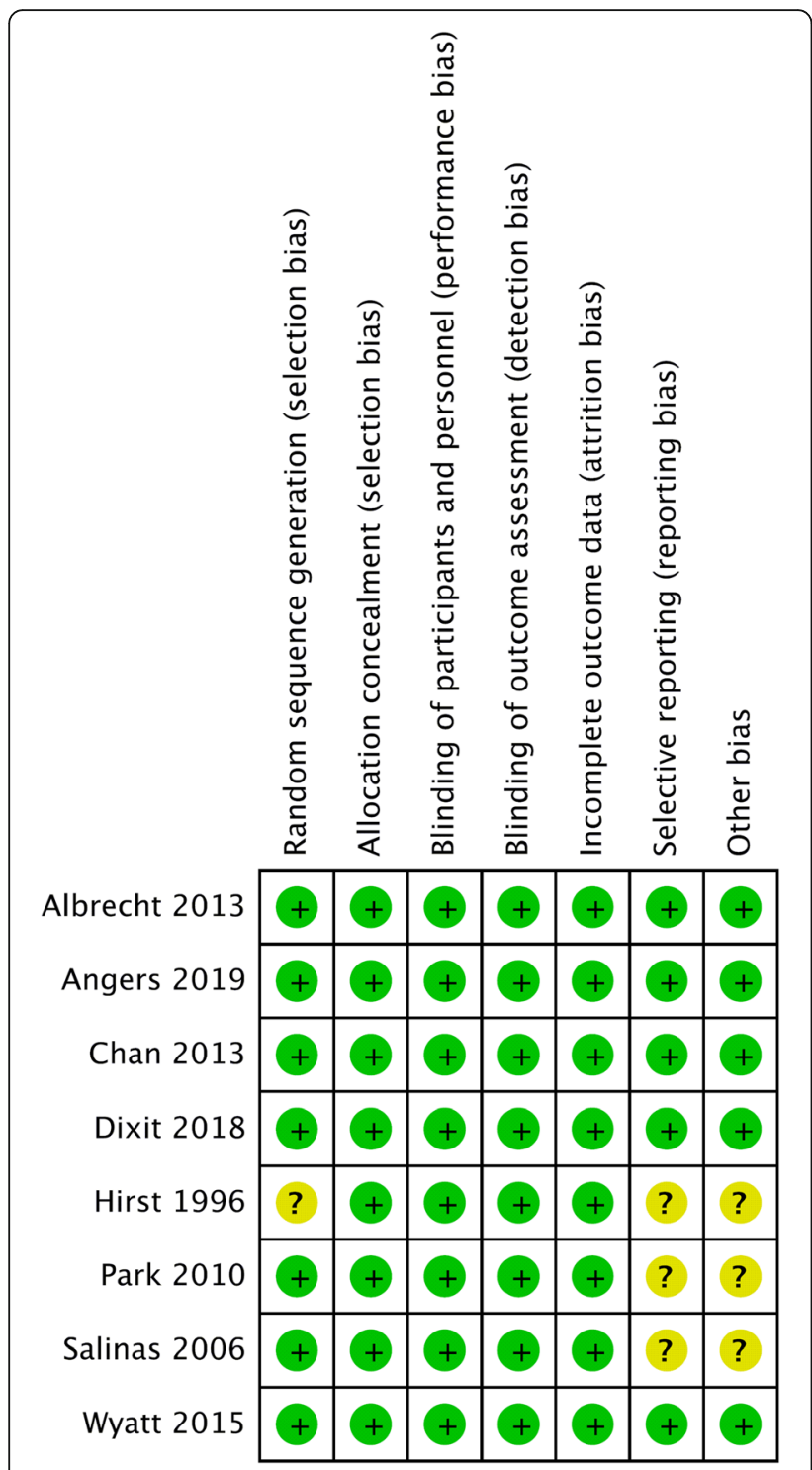

Fig. 9 Results of risk of bias evaluation for the included study

mid-term quadriceps strength, knee range of motion and WOMAC scores. Patients that received PCA alone performed better on these outcome domains compared with patients who received either cFNB or sFNB plus PCA. Interestingly, the effect of decreased quadriceps strength recovery in the cFNB and sFNB group could last up to 12 months [14]. We can only describe the functional outcomes qualitatively rather than to perform a quantitative analysis because of the heterogenous outcome domains used in the studies.

In the current study. cFNB did not lead to a shorter length of hospital stay compared with the sFNB group. Although cFNB was associated with less opioid consumption compared with the sFNB group, this benefit did not seem to translate to a faster functional recovery or a shorter length of hospital stay. In addition, the 5 studies that evaluated duration of hospital stay had different discharge criteria $[10,12,14,18,19]$. The heterogenous discharge criteria could greatly weaken the clinical implication of this parameter.

Postoperative fall has been one of the most common complications in patients receiving either cFNB or sFNB $[25,26]$. Feibel et al. reported a large series of 1190 patients who underwent any form of knee arthroplasty and received cFNB for 2-3 days. Major falls and permanent femoral nerve injury were the most common complications [25]. Turbitt et al. reported a higher rate of falls from a large series of 3736 patients who had received cFNB [27]. As for sFNB, Sharma et al. evaluated 709 patients who received sFNB after TKA surgery. Falls were the most common complication and reoperations were required in 3 patients $(0.4 \%)$. To compare the safety with regard to risk of fall between $\mathrm{CPNB}$ and sPNB, Ilfeld et al. found a higher rate of falls in patients who received a cPNB than patients with sPNB. This raises concerns for the safety of cFNB, especially in the setting of ERAS [28]. Wasserstein et al. evaluated the risk factors of inpatient falls after 2197 primary TKAs. The overall incidence of fall was $2.7 \%$. Advanced age, BMI $>30 \mathrm{~kg} / \mathrm{m}^{2}$ and cFNB were all independent risk factors that were associated with inpatient falls [29]. In our analysis, there were 346 patients included in the cFNB group and 280 in the sFNB group. Only 2 patients in the sFNB group had a fall. According to the reported incidence of fall [25-27], the relatively smaller sample size in this analysis might be insufficient to draw a conclusion on this rare but important adverse event. As stated by some studies, a comprehensive fall prevention care would be warranted to modify and lower the risk of falls after both sFNB and cFNB [12, 18, 30, 31].

There are some limitations that should be recognized. First, we searched only for English articles but not articles in other languages or unpublished data. This would be a potential source of publication bias. Second, there was a high heterogeneity between studies including age, gender, doses and regimens used in $\mathrm{cFNB}$ and sFNB, type of anesthesia and pain management modalities included in the multimodal analgesic protocol. Third, this meta-analysis compared the efficacy of cFNB and sFNB based on outcome domains including pain scores and opioid consumption. However, there is limited data in the studies so we could not validate whether these benefits can lead to enhanced recovery after surgery, improved long-term functional outcome and less total cost. In addition, the relatively small sample size made it difficult to compare the risk of several rare adverse events, especially falls related to the use of cFNB or sFNB. The incidence was evaluated in several case series with large sample size [25-27] but there is lack of RCTs to evaluate this outcome domain. Furthermore, it is interesting that 
Dixit et al. noted both cFNB and sFNB led to a decreased quadriceps strength up to 12 months, compared with the control group [18]. Some studies estimated that the return of quadriceps motor function or proprioception after discontinuation of cFNB were around 3 to 16 h $[27,32,33]$. Further studies should investigate the association of cFNB and sFNB postoperative quadriceps strength and to provide more information to formulate a fall prevention strategy.

\section{Conclusions}

In the setting of a multimodal analgesic protocol, patients might benefit from a cFNB with regards to a reduced consumption of opioids in the early postoperative period. In terms of pain alleviation, both modalities provided substantial pain relief during the postoperative period and no clinically significant differences were noted between the two groups.

\section{Supplementary information}

Supplementary information accompanies this paper at https://doi.org/10. 1186/s12891-020-3148-1.

Additional file 1. Fig. S1. Funnel plot. (VAS score at postoperative $24 \mathrm{~h}$ ).

Additional file 2. Fig. S2. Funnel plot. (VAS score at postoperative 48 h).

Additional file 3. Fig. S3. Funnel plot. (Total amount of opioids consumed at postoperative $24 \mathrm{~h}$ )

Additional file 4. Fig. S4. Funnel plot. (Total amount of opioids consumed at postoperative $48 \mathrm{~h}$.

Additional file 5. Fig. S5. Funnel plot. (Length of hospital stay).

Additional file 6. Fig. S6. Funnel plot. (Postoperative nausea rate).

\section{Abbreviations}

AOR: Adjusted odds ratio; CFNB: Continuous femoral nerve block: ERAS: Enhanced recovery after surgery; MCID: Minimal clinically important difference; PCA: Patient controlled analgesia; PRISMA: Preferred reporting items for systematic reviews and meta-analysis; RCTs: Randomized controlled trials; SFNB: Single-injection femoral nerve block; SMDs: Standardized mean differences; TKA: Total knee arthroplasty; VAS: Visual analog scale

\section{Acknowledgements}

Not applicable

\section{Authors' contributions}

Concept, literature search and data collection: H-HM, S-WT.

Statistics, data analysis and interpretation: H-HM, S-WT, C-FC, P-KW, W-MC Drafting article: H-HM, S-WT, C-FC, W-MC

Critical revision of article: T-FAC, S-WT, C-FC, P-KW, W-MC

All authors have read and approved the manuscript

\section{Funding}

This study received no funding.

\section{Availability of data and materials}

The information to access the data used in the study is included within this article.

\section{Ethics approval and consent to participate}

Not applicable.

\section{Consent for publication}

Not applicable.

\section{Competing interests}

The authors declare that they have no competing interests.

Received: 3 December 2019 Accepted: 19 February 2020

Published online: 24 February 2020

\section{References}

1. Soffin EM, YaDeau JT. Enhanced recovery after surgery for primary hip and knee arthroplasty: a review of the evidence. Br J Anaesth. 2016;117(suppl 3): iii62-72.

2. Kehlet H, Dahl JB. The value of "multimodal" or "balanced analgesia" in postoperative pain treatment. Anesth Analg. 1993;77(5):1048-56.

3. Parvizi J, Bloomfield MR. Multimodal pain management in orthopedics: implications for joint arthroplasty surgery. Orthopedics. 2013;36(2 Suppl):714

4. American Society of Anesthesiologists Task Force on Acute Pain M. Practice guidelines for acute pain management in the perioperative setting: an updated report by the American Society of Anesthesiologists Task Force on acute pain management. Anesthesiology. 2012;1 16(2):248-73.

5. Strassels SA, Chen C, Carr DB. Postoperative analgesia: economics, resource use, and patient satisfaction in an urban teaching hospital. Anesth Analg. 2002:94(1):130-7 table of contents.

6. Ilfeld BM, Le LT, Meyer RS, Mariano ER, Vandenborne K, Duncan PW, Sessler DI, Enneking FK, Shuster JJ, Theriaque DW, et al. Ambulatory continuous femoral nerve blocks decrease time to discharge readiness after tricompartment total knee arthroplasty: a randomized, triple-masked, placebo-controlled study. Anesthesiology. 2008;108(4):703-13.

7. Ilfeld BM, Mariano ER, Girard PJ, Loland VJ, Meyer RS, Donovan JF, Pugh GA, Le LT, Sessler DI, Shuster JJ, et al. A multicenter, randomized, triple-masked, placebo-controlled trial of the effect of ambulatory continuous femoral nerve blocks on discharge-readiness following total knee arthroplasty in patients on general orthopaedic wards. Pain. 2010;150(3):477-84.

8. Iffeld BM, Shuster JJ, Theriaque DW, Mariano ER, Girard PJ, Loland VJ, Meyer S, Donovan JF, Pugh GA, Le LT, et al. Long-term pain, stiffness, and functional disability after total knee arthroplasty with and without an extended ambulatory continuous femoral nerve block: a prospective, 1-year follow-up of a multicenter, randomized, triple-masked, placebo-controlled trial. Reg Anesth Pain Med. 2011;36(2):116-20.

9. Hirst GC, Lang SA, Dust WN, Cassidy JD, Yip RW. Femoral nerve block. Single injection versus continuous infusion for total knee arthroplasty. Reg Anesth. 1996;21(4):292-7.

10. Salinas FV, Liu SS, Mulroy MF. The effect of single-injection femoral nerve block versus continuous femoral nerve block after total knee arthroplasty on hospital length of stay and long-term functional recovery within an established clinical pathway. Anesth Analg. 2006;102(4):1234-9.

11. Park CK, Cho CK, Lee GG, Lee JH. Optimizing dose infusion of $0.125 \%$ bupivacaine for continuous femoral nerve block after total knee replacement. Korean J Anesthesiol. 2010:58(5):468-76.

12. Chan EY, Fransen M, Sathappan S, Chua NH, Chan YH, Chua N. Comparing the analgesia effects of single-injection and continuous femoral nerve blocks with patient controlled analgesia after total knee arthroplasty. J Arthroplast. 2013;28(4):608-13.

13. Chan EY, Fransen M, Parker DA, Assam PN, Chua N. Femoral nerve blocks for acute postoperative pain after knee replacement surgery. Cochrane Database Syst Rev. 2014:5:CD009941.

14. Angers M, Belzile EL, Vachon J, Beauchamp-Chalifour P, Pelet S. Negative influence of femoral nerve block on quadriceps strength recovery following total knee replacement: a prospective randomized trial. Orthop Traumatol Surg Res. 2019:105(4):633-7.

15. Ma H-H, Chou T-FA, Tsai S-W, Chen C-F, Wu P-K, Chen W-M. The efficacy of intraoperative periarticular injection in Total hip arthroplasty: a systematic review and meta-analysis. BMC Musculoskelet Disord. 2019;20(1):269.

16. Danoff JR, Goel R, Sutton R, Maltenfort MG, Austin MS. How much pain is significant? Defining the minimal clinically important difference for the visual analog scale for pain after Total joint Arthroplasty. J Arthroplast. 2018; 33(75):S71-5 e72.

17. Albrecht E, Morfey D, Chan V, Gandhi R, Koshkin A, Chin K, Robinson S, Frascarolo P, Brull R. Single-injection or continuous femoral nerve block for total knee arthroplasty? Clin Orthop Relat Res. 2014;472(5):1384-93.

18. Dixit V, Fathima S, Walsh SM, Seviciu A, Schwendt I, Spittler K-H, Briggs D. Effectiveness of continuous versus single injection femoral nerve block for 
total knee arthroplasty: a double blinded, randomized trial. Knee. 2018;25(4): 623-30

19. Wyatt MC, Wright T, Locker J, Stout K, Chapple C, Theis JC. Femoral nerve infusion after primary total knee arthroplasty: a prospective, double-blind, randomised and placebo-controlled trial. Bone Joint Res. 2015;4(2):11-6.

20. Szczukowski MJ Jr, Hines JA, Snell JA, Sisca TS. Femoral nerve block for total knee arthroplasty patients: a method to control postoperative pain. J Arthroplast. 2004;19(6):720-5.

21. Allen HW, Liu SS, Ware PD, Nairn CS, Owens BD. Peripheral nerve blocks improve analgesia after total knee replacement surgery. Anesth Analg. 1998; 87(1):93-7.

22. Helmerhorst GT, Teunis T, Janssen SJ, Ring D. An epidemic of the use, misuse and overdose of opioids and deaths due to overdose, in the United States and Canada: is Europe next? Bone Joint J. 2017;99-B(7):856-64.

23. Clark DJ, Schumacher MA. America's opioid epidemic: supply and demand considerations. Anesth Analg. 2017;125(5):1667-74.

24. Trasolini NA, McKnight BM, Dorr LD. The opioid crisis and the orthopedic surgeon. J Arthroplast. 2018;33(11):3379-82 e3371.

25. Feibel RJ, Dervin GF, Kim PR, Beaule PE. Major complications associated with femoral nerve catheters for knee arthroplasty: a word of caution. J Arthroplast. 2009;24(6 Suppl):132-7.

26. Sharma S, lorio R, Specht LM, Davies-Lepie S, Healy WL. Complications of femoral nerve block for total knee arthroplasty. Clin Orthop Relat Res. 2010; 468(1):135-40

27. Turbitt LR, MCHardy PG, Casanova M, Shapiro J, Li L, Choi S. Analysis of inpatient falls after Total knee Arthroplasty in patients with continuous femoral nerve block. Anesth Analg. 2018;127(1):224-7.

28. Iffeld BM, Duke KB, Donohue MC. The association between lower extremity continuous peripheral nerve blocks and patient falls after knee and hip arthroplasty. Anesth Analg. 2010;111(6):1552-4

29. Wasserstein D, Farlinger C, Brull R, Mahomed N, Gandhi R. Advanced age, obesity and continuous femoral nerve blockade are independent risk factors for inpatient falls after primary total knee arthroplasty. J Arthroplast. 2013;28(7):1121-4.

30. Ilfeld BM. Single-injection and continuous femoral nerve blocks are associated with different risks of falling. Anesthesiology. 2014;121(3):668-9.

31. Memtsoudis SG, Danninger T, Rasul R, Poeran J, Gerner P, Stundner O, Mariano ER, Mazumdar M. Inpatient falls after total knee arthroplasty: the role of anesthesia type and peripheral nerve blocks. Anesthesiology. 2014; 120(3):551-63.

32. Iffeld BM, Loland VJ, Sandhu NS, Suresh PJ, Bishop MJ, Donohue MC Ferguson EJ, Madison SJ. Continuous femoral nerve blocks: the impact of catheter tip location relative to the femoral nerve (anterior versus posterior) on quadriceps weakness and cutaneous sensory block. Anesth Analg. 2012; 115(3):721-7.

33. Charous MT, Madison SJ, Suresh PJ, Sandhu NS, Loland VJ, Mariano ER, Donohue MC, Dutton PH, Ferguson EJ, Ilfeld BM. Continuous femoral nerve blocks: varying local anesthetic delivery method (bolus versus basal) to minimize quadriceps motor block while maintaining sensory block. Anesthesiology. 2011:115(4):774-81.

\section{Publisher's Note}

Springer Nature remains neutral with regard to jurisdictional claims in published maps and institutional affiliations.

Ready to submit your research? Choose BMC and benefit from:

- fast, convenient online submission

- thorough peer review by experienced researchers in your field

- rapid publication on acceptance

- support for research data, including large and complex data types

- gold Open Access which fosters wider collaboration and increased citations

- maximum visibility for your research: over $100 \mathrm{M}$ website views per year

At BMC, research is always in progress.

Learn more biomedcentral.com/submissions 